\title{
Rhabdomyolysis-Induced Acute Renal Failure Following Fenofibrate Therapy: A Case Report and Literature Review
}

\author{
Ramazan Danis, ${ }^{1}$ Sami Akbulut, ${ }^{2}$ Sehmus Ozmen, ${ }^{1}$ and Senay Arikan ${ }^{3}$ \\ ${ }^{1}$ Department of Nephrology, Diyarbakir Education and Research Hospital, 21400 Diyarbakir, Turkey \\ ${ }^{2}$ Department of Surgery, Diyarbakir Education and Research Hospital, 21400 Diyarbakir, Turkey \\ ${ }^{3}$ Department of Endocrinology and Metabolism, Faculty of Medicine, Dicle Universiy, 21280 Diyarbakir, Turkey
}

Correspondence should be addressed to Sami Akbulut, akbulutsami@gmail.com

Received 11 May 2010; Accepted 25 June 2010

Academic Editor: M. E. Safar

Copyright ( 2010 Ramazan Danis et al. This is an open access article distributed under the Creative Commons Attribution License, which permits unrestricted use, distribution, and reproduction in any medium, provided the original work is properly cited.

Fenofibrate, a fibric acid derivative, is used to treat diabetic dyslipidemia, hypertriglyceridemia, and combined hyperlipidemia, administered alone or in combination with statins. Rhabdomyolysis is defined as a pathological condition involving skeletal muscle cell damage leading to the release of toxic intracellular material into circulation. Its major causes include muscle compression or overexertion; trauma; ischemia; toxins; cocaine, alcohol, and drug use; metabolic disorders; infections. However, rhabdomyolysis associated with fenofibrate is extremely rare. Herein we report a 45-year-old female patient who was referred to our department because of generalized muscle pain, fatigue, weakness, and oliguria over the preceding 3 weeks. On the basis of the pathogenesis and clinical and laboratory examinations, a diagnosis of acute renal failure secondary to fenofibrate-induced rhabdomyolysis was made. Weekly followups for patients who are administered fenofibrate are the most important way to prevent possible complications.

\section{Introduction}

Rhabdomyolysis is a clinical and biochemical syndrome resulting from skeletal muscle injury and the consequent release of muscle cell constituents into circulation. It may result in myoglobinuria, the filtration of myoglobin into the urine, and is often associated with acute renal failure (ARF) [1]. Its major causes include muscle compression or overexertion; ischemia; toxins; metabolic disorders; cocaine [2], alcohol [2], and drug use; infections [3]. However, druginduced rhabdomyolysis occurs rarely. In nearly all presented cases, a predisposing factor for rhabdomyolysis, such as high statin dose, diabetes, older age, female gender, renal disease, or hypothyroidism, is present $[4,5]$. Herein, we report a 45year-old diabetic female patient without any known prior renal disease who presented with acute renal failure that developed after fenofibrate treatment.

\section{Case Report}

A 45-year-old female patient presented to our hospital with complaints of muscle pain, weakness, fatigue, decreased urine outflow, and a dark brown urine color for the previous 3 weeks. She had a long history of type 2 diabetes and had had hypertriglyceridemia for about 2 years, which was treated with glimepiride ( $3 \mathrm{mg}$ daily) and metformin (2 1 -g tablets daily). About 3 weeks before presentation, she was prescribed fenofibrate $200 \mathrm{mg}$ daily for hypertriglyceridemia, and she had used it regularly. Her history included diabetic hypertension treated uneventfully with perindopril for 2 years. She had no family history of liver, muscle, or kidney disease, had not traveled recently, and was sexually stable with no history of alcohol, tobacco, or drug abuse. Decreased urine output and generalized weakness (3/5, muscle strength) and muscular tenderness were detected on clinical examination. Laboratory investigations revealed the following levels: serum creatine kinase (CK): 5698 U/L; AST: 179 U/L; ALT: $191 \mathrm{U} / \mathrm{L}$; creatinine: $2.2 \mathrm{mg} / \mathrm{dL}$; urea: $150 \mathrm{mg} / \mathrm{dL}$; LDH: $974 \mathrm{U} / \mathrm{L}$; fasting glucose: $190 \mathrm{mg} / \mathrm{dL}$; HbA1C: $7.85 \%$; triglyceride: $177 \mathrm{mg} / \mathrm{dL}$; total cholesterol: $164 \mathrm{mg} / \mathrm{dL}$; myoglobulin: $>100,000 \mathrm{mcg} / \mathrm{ml}$. Her liver and renal functions tests were normal before the fenofibrate therapy. She had no recent viral illness, history of trauma, epilepsy, hypothyroidism, or over-the-counter medication 
TABLE 1: Results of our patient's initial, second day, and last day laboratory tests.

\begin{tabular}{lcccc}
\hline Serum & Initial day & Second day & Third day & Reference range \\
\hline Urea $(\mathrm{mg} / \mathrm{dL})$ & 29.5 & 20.3 & 12.2 & $10-45$ \\
Creatinine $(\mathrm{mg} / \mathrm{dL})$ & 2.2 & 1.4 & 31.3 & $0.5-1.14$ \\
ALT $(\mathrm{U} / \mathrm{L})$ & 9.7 & 18.3 & 24.2 & $10-35$ \\
AST $(\mathrm{U} / \mathrm{L})$ & 27.6 & 25.2 & 19.9 & $10-40$ \\
$\mathrm{CK}(\mathrm{U} / \mathrm{L})$ & 7.8 & 28.7 & 17.10 & $38-174$ \\
$\mathrm{LDH}(\mathrm{U} / \mathrm{L})$ & 31.8 & 23.11 & 19.5 & $125-243$ \\
$\mathrm{Na}(\mathrm{mmol} / \mathrm{L})$ & 18.5 & 22.5 & 8.9 & $136-145$ \\
$\mathrm{Ca}(\mathrm{mg} / \mathrm{dL})$ & 8.0 & 8.4 & 3.8 & $8.4-10.2$ \\
$\mathrm{~K}(\mathrm{mmol} / \mathrm{L})$ & 3.9 & 3.6 & 2.3 & $3.5-5.1$ \\
$\mathrm{P}(\mathrm{mg} / \mathrm{dL})$ & 3.4 & 2.6 & & $2.7-4.5$ \\
\hline
\end{tabular}

TABLE 2: A summary of reported fifteen cases of rhabdomyolysis associated with fenofibrate therapy.

\begin{tabular}{|c|c|c|c|c|c|c|c|c|c|c|}
\hline & References & Year & Age & Sex & Medical History & Other drug & Fenofibrate & BUN & $\mathrm{Cr}$ & CK \\
\hline 1 & Wu et al. [6] & 2009 & 52 & $\mathrm{~F}$ & $\mathrm{DL}$ & & $200 \mathrm{mg}$, for $1 \mathrm{mo}$ & 43.8 & & \\
\hline 2 & De Souza et al. [7] & 2009 & 54 & $\mathrm{M}$ & CRF, DL, HT, Hypothyroidism & & $200 \mathrm{mg}$, for $2 \mathrm{mo}$ & 120 & 4.9 & 52749 \\
\hline 3 & Çetinkaya et al. [5] & 2008 & 60 & $\mathrm{~F}$ & DM-II, HT, DL & & $200 \mathrm{mg}$, for NA & & 4.2 & 11867 \\
\hline \multirow{2}{*}{4} & \multirow{2}{*}{ Unal et al. [1] } & \multirow{2}{*}{2008} & 56 & F & CAD, DL & Pravastatin & $200 \mathrm{mg}$, for $2 \mathrm{mo}$ & 37 & 2.6 & 97392 \\
\hline & & & 58 & M & CABG, DL & Atorvastatin & $200 \mathrm{mg}$, for $1 \mathrm{mo}$ & 36 & 3.6 & 96639 \\
\hline 5 & Yildiz et al. [8] & 2008 & 74 & M & CABG, HT, DL & & $267 \mathrm{mg}$, for $2 \mathrm{wk}$ & 224 & 5.3 & 26680 \\
\hline 6 & Tahmaz et al. [9] & 2007 & 42 & $\mathrm{~F}$ & HT, DL & & $250 \mathrm{mg}$, for $4 \mathrm{wk}$ & 90 & 5.5 & 21000 \\
\hline 7 & Dedhia and Munsi [10] & 2007 & 68 & $\mathrm{M}$ & CABGx2, HT, DL & Rosuvastatin & $160 \mathrm{mg}$, for $3 \mathrm{wk}$ & & 2.3 & 23665 \\
\hline 8 & Jacob et al. [11] & 2005 & 70 & $\mathrm{M}$ & DM-II, HT, DL, Hypothyroidism & Simvastatin & $160 \mathrm{mg}$, for $4 \mathrm{wk}$ & & 2.7 & 10936 \\
\hline 9 & Ireland et al. [12] & 2005 & 67 & $\mathrm{~F}$ & HT, DL & Rosuvastatin & $160 \mathrm{mg}$, for $2 \mathrm{wk}$ & 58 & 3.6 & 13808 \\
\hline 10 & Kursat et al. [13] & 2005 & 63 & $\mathrm{~F}$ & HT, DL, Hypothyroidism & Simvastatin & $200 \mathrm{mg}$, for $4 \mathrm{wk}$ & 188 & 4.5 & 8842 \\
\hline 11 & Ghosh et al. [14] & 2004 & 58 & M & $\mathrm{CAD}, \mathrm{DL}$ & & $200 \mathrm{mg}$, for $5 \mathrm{wk}$ & & & 1129 \\
\hline 12 & Barker et al. [15] & 2003 & 56 & $\mathrm{~F}$ & HT, DM-II, DL & & $200 \mathrm{mg}$, for $10 \mathrm{~d}$ & & 2 & 5632 \\
\hline \multirow{2}{*}{13} & \multirow{2}{*}{ Clouatre et al. [16] } & \multirow{2}{*}{1999} & 57 & $\mathrm{~F}$ & CRF, DL, HT, Hypothyroidism & & $200 \mathrm{mg}$, for $4 \mathrm{wk}$ & & & 8850 \\
\hline & & & 55 & $\mathrm{~F}$ & CRF, PCRD, DL, Hypothyroidism & Simvastatin & $200 \mathrm{mg}$, for $3 \mathrm{wk}$ & & & 11360 \\
\hline
\end{tabular}

BUN: blood urea nitrogen $(\mathrm{mg} / \mathrm{dl}), \mathrm{Cr}$ : creatinine $(\mathrm{mg} / \mathrm{dl})$, DM: diabetes mellitus, CRF: chronic renal failure, CK: serum creatine kinase (IU/L), HT: hypertension, DL: dyslipidemia, CAD: coronary artery disease, CABG: coronary artery by-pass graft, and PCRD: polycystic renal disease.

use and had not taken any other medication known to induce rhabdomyolysis. The fenofibrate was discontinued, and intravenous fluid replacement with bicarbonate therapy was started. The myalgia resolved, urine output was normalized, and serum urea and creatinine decreased to normal values on the second day of treatment. The initial, second, and last day laboratory results are presented in Table 1.

\section{Discussion}

Fenofibrate is a derivative of fibric acid. It reduces very low-density lipoprotein (VLDL) and low-density lipoprotein (LDL) and increases high-density lipoprotein (HDL). The side effects of fibrate treatment include gastrointestinal complaints, gallstones, skin reactions, and blood disturbances that are tolerable and reversible. The most important side effect of fenofibrate is rhabdomyolysis $[1,5]$. Rhabdomyolysis is a syndrome characterized by muscle necrosis and the release of intracellular muscle contents into systemic circu- lation. Oliguric or nonoliguric ARF is the most common complication of rhabdomyolysis, occurring in $10 \%-40 \%$ of patients [17].

We established a diagnosis of ARF, which was probably due to fenofibrate-induced rhabdomyolysis based on elevated CK and the absence of any other risk factor related to rhabdomyolysis and ARF according to the Naranjo probability scale [18]. The demographics, medical history, and personal history of this patient did not suggest any underlying disease that may have caused the rhabdomyolysis; however, the time sequence of the start of the fenofibrate and onset of the ARF were consistent with drug-related rhabdomyolysisrelated renal injury. Although no rechallenge was attempted, the rapid improvement in muscular enzyme levels and renal function after discontinuation of fenofibrate suggests an association with ARF and rhabdomyolysis related to the use of the drug. Other causes for muscular and/or renal injury (e.g., viruses, toxic exposures, or diabetes mellitus) were possible but not suggested by the clinical history. In our case, rapidly diminishing enzymes on the third day after stopping 
the fenofibrate therapy indicated that the current clinical situation was associated with fenofibrate.

Acute renal failure secondary to fenofibrate monotherapy-induced rhabdomyolysis is a rare and newly encountered clinical condition, and there are only sixteen cases associated with fenofibrate therapy (nine with monotherapy and seven with fenofibrate-statin combination therapy) which have been published in the English-language literature. Fifteen of them are summarized in Table $2[1,4-16]$. The risk for serious muscle toxicity appears to be increased in elderly patients and in patients with diabetes $[5,11,15]$, renal failure $[4,7,16]$, or hypothyroidism $[4,5,7,11,13,15,16]$. For the patient we present here, tests done 2 months previously found normal results for renal function, thyroid function, and anti-TPO antibody levels. The only risk factor she had was type 2 diabetes mellitus.

Çetinkaya et al. [5] indicated that dehydration can be an additive risk factor in elderly patients who use fenofibrate; thus, patients in this group should be carefully informed about possible side effects and should be followed closely during the therapy. The patient presented here did not come to any of the controls after starting the therapy, and when she finally came, she had moderate dehydration.

In conclusion, prescribers and users of fenofibrate should be alert to the possibility of its potentially fatal side effects such as rhabdomyolysis and related ARF. Screening for risk factors such as hepatic impairment, renal insufficiency, serious infections, hypothyroidism, and diabetes should also be considered, especially in elderly patients who are treated with fenofibrate. Doctors who work in regions in which doctor-patient relationships are poor should exercise particular caution when prescribing such drugs.

\section{Competing Interests}

The authors declare that they have no competing interests.

\section{Authors' Contribution}

R. Danis, S. Akbulut, and S. Ozmen contributed in writing the paper and review of the literature as well as undertaking a comprehensive literature search; S. Akbulut and S. Arikan contributed to the paper design and preparation.

\section{References}

[1] A. Unal, E. Torun, M. H. Sipahioglu et al., "Fenofibrateinduced acute renal failure due to massive rhabdomyolysis after coadministration of statin in two patients," Internal Medicine, vol. 47, no. 11, pp. 1017-1019, 2008.

[2] L. Alconcher, M. B. Meneguzzi, G. Rudolf, and F. Criado, "Rhabdomyolisis associated with alcohol and cocaine intake," Archivos Argentinos de Pediatria, vol. 106, no. 5, pp. 454-457, 2008.

[3] G. Melli, V. Chaudhry, and D. R. Cornblath, "Rhabdomyolysis: an evaluation of 475 hospitalized patients," Medicine, vol. 84, no. 6, pp. 377-385, 2005.

[4] R. L. Satarasinghe, R. Ramesh, A. A. A. Riyaaz, P. A. K. G. Gunarathne, and A. P. de Silva, "Hypothyroidism is a predisposing factor for fenofibrate-induced rhabdomyolysispatient report and literature review," Drug Metabolism and Drug Interactions, vol. 22, no. 4, pp. 279-283, 2007.

[5] R. Çetinkaya, A. Uyanik, R. Yildirim, Y. Bilen, and M. Keleş, "Fenofibrate monotherapy-induced rhabdomyolysis in a patient with type-2 diabetes," Indian Journal of Medical Sciences, vol. 62, no. 11, pp. 458-459, 2008.

[6] J. Wu, Y. Song, H. Li, and J. Chen, "Rhabdomyolysis associated with fibrate therapy: review of 76 published cases and a new case report," European Journal of Clinical Pharmacology, vol. 65, no. 12, pp. 1169-1174, 2009.

[7] A. A. de Sousa, H. S. Kronit, F. A. R. Neves, and A. A. Amato, "Fenofibrate-induced rhabdomyolysis in a patient with chronic kidney disease: an unusual presenting feature of hypothyroidism," Arquivos Brasileiros de Endocrinologia e Metabologia, vol. 53, no. 3, pp. 383-386, 2009.

[8] A. Yildiz, E. Gucuk, and S. Cay, "A case of rhabdomyolysis and thromboembolic event secondary to fibrate monotherapy," Acta Cardiologica, vol. 63, no. 4, pp. 515-517, 2008.

[9] M. Tahmaz, B. Kumbasar, K. Ergen, U. Ure, G. Karatemiz, and R. Kazancioglu, "Acute renal failure secondary to fenofibrate monotherapy-induced rhabdomyolysis," Renal Failure, vol. 29, no. 7, pp. 927-930, 2007.

[10] V. Dedhia and S. C. Munsi, "Myopathy caused by a combination rosuvastatin and fenofibrate," Journal of Association of Physicians of India, vol. 55, pp. 152-153, 2007.

[11] S. S. Jacob, S. Jacob, C. Williams, and M. A. Deeg, "Simvastatin, fenofibrate, and rhabdomyolysis," Diabetes Care, vol. 28, no. 5, p. 1258, 2005.

[12] J. H. E. Ireland, C. H. Eggert, C. J. Arendt, and A. W. Williams, "Rhabdomyolysis with cardiac involvement and acute renal failure in a patient taking rosuvastatin and fenofibrate," Annals of Internal Medicine, vol. 142, no. 11, pp. 949-950, 2005.

[13] S. Kursat, T. Alici, and H. B. Colak, "A case of rhabdomyolysis induced acute renal failure secondary to statin-fibratederivative combination and occult hypothyroidism," Clinical Nephrology, vol. 64, no. 5, pp. 391-393, 2005.

[14] B. Ghosh, S. Sengupta, B. Bhattacharjee, A. Majumder, and S. B. Sarkar, "Fenofibrate-induced myopathy," Neurology India, vol. 52, no. 2, pp. 268-269, 2004.

[15] B. J. Barker, R. R. Goodenough, and J. M. Falko, "Fenofibrate monotherapy induced rhabdomyolysis," Diabetes Care, vol. 26, no. 8, pp. 2482-2483, 2003.

[16] Y. Clouatre, M. Leblanc, D. Ouimet, and V. Pichette, "Fenofibrate-induced rhabdomyolysis in two dialysis patients with hypothyroidism," Nephrology Dialysis Transplantation, vol. 14, no. 4, pp. 1047-1048, 1999.

[17] R. Vanholder, M. S. Sever, E. Erek, and N. Lameire, "Rhabdomyolysis," Journal of the American Society of Nephrology, vol. 11, no. 8, pp. 1553-1561, 2000.

[18] C. A. Naranjo, U. Busto, and E. M. Sellers, "A method for estimating the probability of adverse drug reactions," Clinical Pharmacology and Therapeutics, vol. 30, no. 2, pp. 239-245, 1981. 


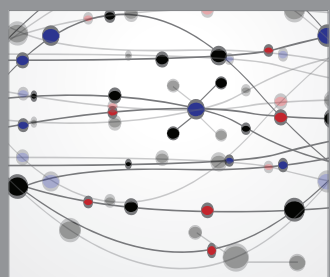

The Scientific World Journal
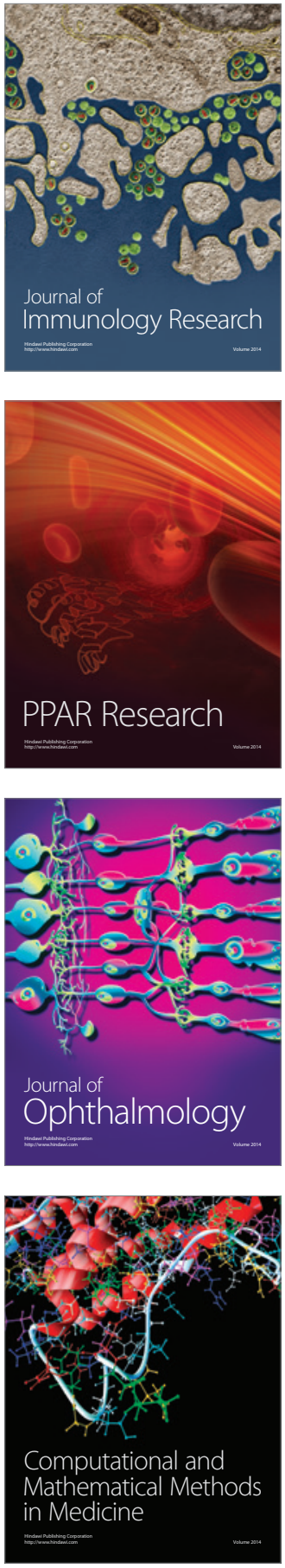

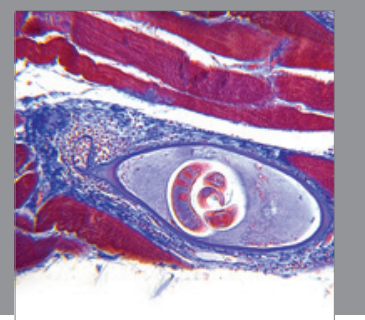

Gastroenterology

Research and Practice
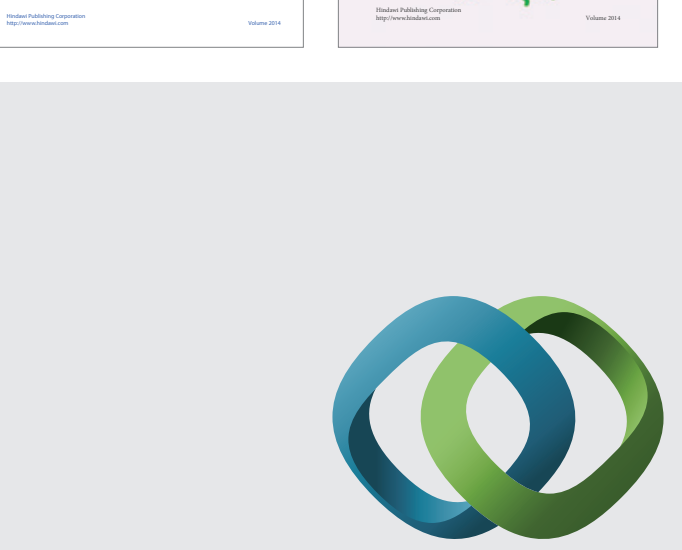

\section{Hindawi}

Submit your manuscripts at

http://www.hindawi.com
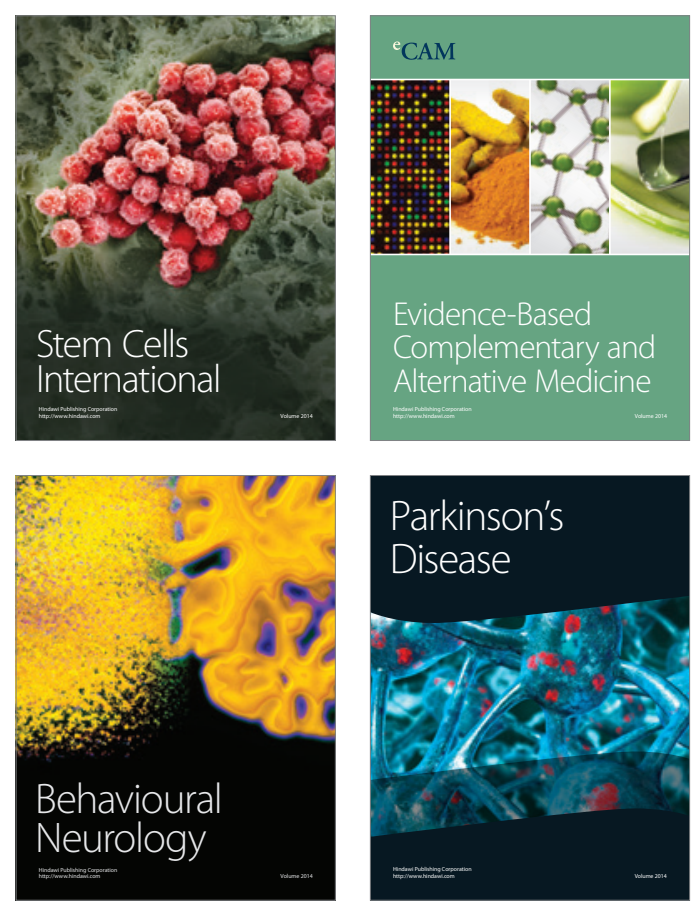

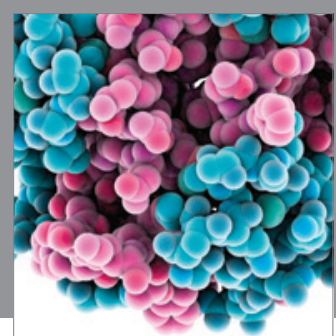

Journal of
Diabetes Research

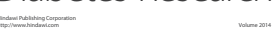

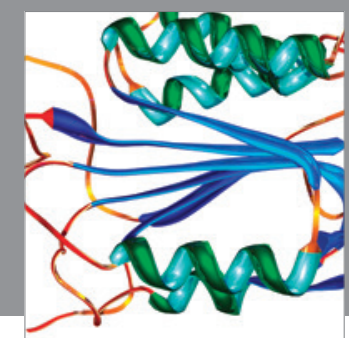

Disease Markers
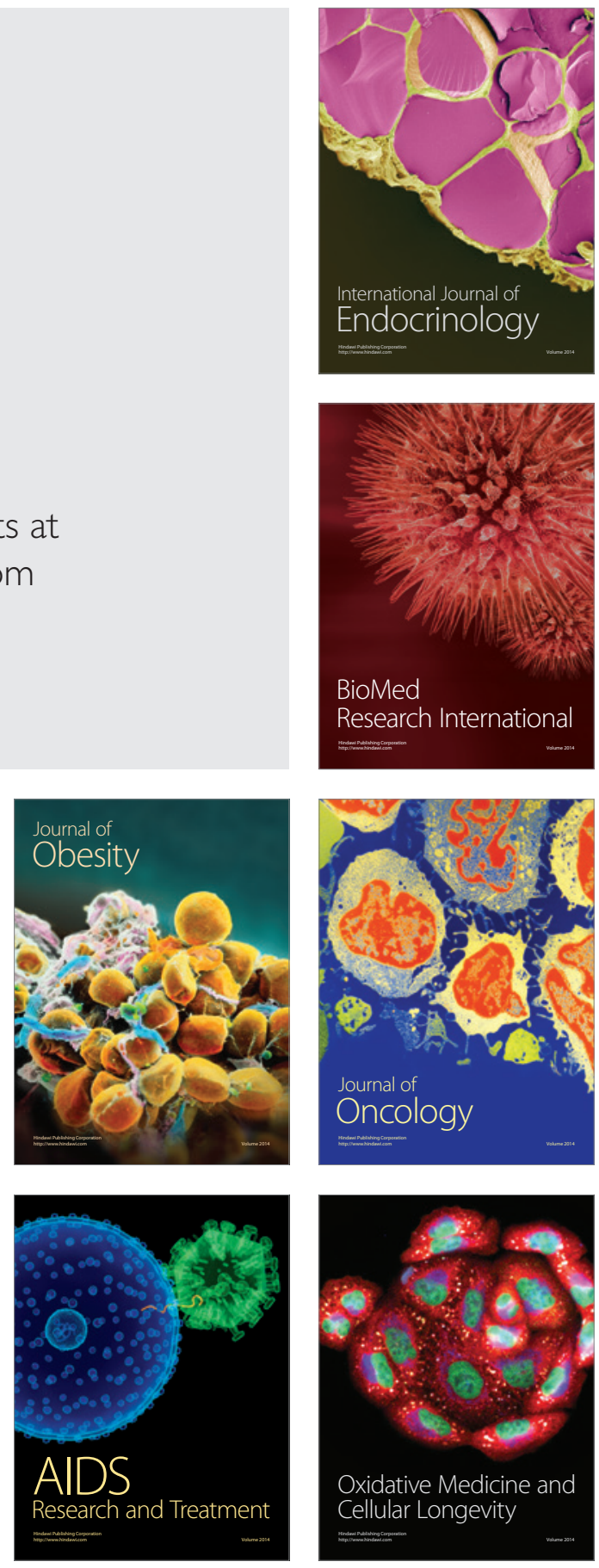